\title{
Investigating Spherical Images of a Curve According to Type-1 Bishop Frame in Weyl Space Using Prolonged Covariant Derivative
}

\author{
Nil Kofoğlu $1^{*}$ \\ $1^{*}$ Department of Software Engineering, Faculty of Engineering and Architecture, Beykent University, 34398, İstanbul, Turkey, (ORCID: 0000-0003-4361-3555), \\ nilkofoglu@beykent.edu.tr
}

(First received 7 July 2021 and in final form 13 September 2021)

(DOI: $10.31590 /$ ejosat.963679)

ATIF/REFERENCE: Kofoğlu, N. (2021). Investigating Spherical Images of a Curve According to Type-1 Bishop Frame in Weyl Space Using Prolonged Covariant Derivative. European Journal of Science and Technology, (27), 450-458.

\begin{abstract}
In this study, we investigated spherical images of a curve according to type-1 Bishop frame in three dimensional Weyl space. Further, we expressed the relations among Frenet-Serret and type-1 Bishop frame apparatus. We defined the concepts of general helix, slant helix, spherical curve and also circle by using prolonged covariant derivative in Weyl space. Later, provided that these spherical images satisfy the above definitions, the conditions obtained were expressed in terms of first and second curvatures and hence Bishop curvatures. Additionally, parallel displacement condition of the binormal vector fields of the $n_{1}$ and $\underset{2}{n}$ Bishop spherical images of a curve along their own tangent vector fields was discussed.
\end{abstract}

Keywords: Weyl space, General helix, Slant helix, Spherical curve, Spherical image.

\section{Prolonged Kovaryant Türevi Kullanarak Weyl uzayındaki Bir Eğrinin Tip-1 Bishop Çatısına Göre Küresel Resimlerinin İncelenmesi}

$\ddot{O} \mathbf{z}$

Bu çalışmada, Weyl uzayındaki bir eğrinin tip-1 Bishop çatısına göre küresel resimlerini inceledik. Ayrıca, Frenet-Serret ve tip-1 Bishop çatı aparatları arasındaki bağıntıları ifade ettik. Prolonged kovaryant türevi kullanarak, Weyl uzayında genel helis, slant helis, küresel eğri ve ayrıca çember kavramlarını tanımladık. Daha sonra, bu küresel resimlerin yukarıdaki tanımları sağlaması halinde, elde edilen şartlar birinci ve ikinci eğrilikler ve dolayısıyla Bishop eğrilikleri cinsinden ifade edildi. Bunlara ek olarak, bir eğrinin $n_{1}$ ve ${\underset{2}{2}}_{\text {Bishop }}$ küresel resimlerinin binormal vektör alanlarının kendi teğet vektör alanları boyunca paralel kayma şartı ele alındı.

Anahtar Kelimeler: Weyl uzayı, Genel helis, Slant helis, Küresel eğri, Küresel resim.

\footnotetext{
* Corresponding Author: nilkofoglu@,beykent.edu.tr
} 


\section{Introduction}

Bishop frame (or type-1 Bishop frame) was introduced by Bishop (1975). This frame was also named as alternative or parallel frame of the curves. Many researchers used Bishop frame in several spaces, such as Bükçü and Karacan (2008a and 2009), Yılmaz et al. (2010; in Euclidean space), Bükçü and Karacan (2008b; in Lorentzian space), Karacan and Bükçü (2007 and 2008), Y1lmaz (2009; in Minkowski 3-space) and Kofoğlu (2020; in Weyl space).

\section{Preliminaries}

Let $C: x^{i}=x^{i}(s)(s$ is the arc length parameter of $C$ ) be a curve in three dimensional Weyl space $W_{3}(i=1,2,3)$. Let us denote Frenet-Serret frame and Bishop (or type-1 Bishop) frame belonging to $C$ by $\left\{v_{1}, v_{2}, v\right\}$ and $\left\{v_{1}, n_{1}, n_{2}\right\}$, respectively. Both of these frames are orthonormal bases.

Frenet-Serret formulas are expressed as

$$
\begin{aligned}
& v_{1}^{k} \dot{\nabla}_{k} v_{1}^{i}=\kappa_{1} v_{2}^{i} \\
& v_{1}^{k} \dot{\nabla}_{k} v_{2}^{i}=-\kappa_{1} v_{1}^{i}+\kappa_{2} v_{3}^{v^{i}} \\
& v_{1}^{k} \dot{\nabla}_{k} v_{3}^{i}=-\kappa_{2}{\underset{2}{i}}^{i}
\end{aligned}
$$

where $\kappa_{1}$ and $\kappa_{2}$ are the first and second curvatures of $C$, respectively.

Derivative formulas of the vector fields of Bishop frame are in the following form:

$$
\begin{gathered}
\underset{1}{v^{k}} \dot{\nabla}_{k} v_{1}^{i}=k_{1} \underset{1}{n^{i}}+k_{2} \underset{2}{n^{i}} \\
{\underset{1}{v}}_{\dot{\nabla}_{k}}^{\boldsymbol{n}_{1}^{i}}=-k_{1} \underset{1}{v^{i}} \\
\underset{1}{v^{k}} \dot{\nabla}_{k}{\underset{2}{n}}^{i}=-k_{2} \underset{1}{v^{i}}
\end{gathered}
$$

Here, $k_{1}$ and $k_{2}$ are Bishop curvatures (Bishop, 1975). Their equivalents in Weyl space (Kofoğlu, 2020) are

$$
k_{1}=\stackrel{p}{T}_{k}{ }_{1}^{v^{k}} \underset{p}{v_{1}^{i}}{ }_{1}^{j} g_{i j}(j, k, p=1,2,3)
$$

or

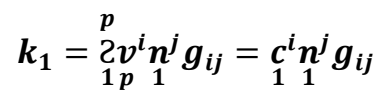

and

$$
k_{2}=\stackrel{p}{T}_{1} \underset{1}{v^{k}} \underset{p}{v_{2}^{i}}{\underset{n}{j}}^{j} g_{i j}
$$

or

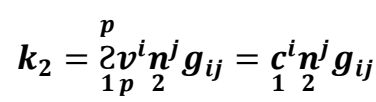

where, $\begin{aligned} & p \\ & 2\end{aligned}=T_{1}^{p} k_{1} v^{k}$ is geodesic curvature of the net $\left(\underset{1}{v}, \begin{array}{c}v \\ 2\end{array}, v\right)$ (Tsareva, 1990) and $c_{1}^{i}=\underset{1 p}{2} v^{i}$ is the geodesic curvature vector field of the net $\left(v_{1}, v_{2}, v\right)$ (Tsareva, 1990).

Also, $k_{1}=\kappa_{1} \cos \theta, \quad k_{2}=\kappa_{1} \sin \theta, \kappa_{1}^{2}=k_{1}^{2}+k_{2}^{2}$ and $\kappa_{2}=$ $v_{1}^{k} \dot{\nabla}_{k} \theta(\theta=\theta(s))$ where $\theta=\Varangle\left(\underset{2}{v^{i}}, n_{1}^{i}\right)($ Kofoğlu, 2020).

There is the following relation among the vector fields of these two frames (Kofoğlu, 2020):

$$
\left(\begin{array}{l}
v^{i} \\
1 \\
v^{i} \\
2 \\
v^{i} \\
3
\end{array}\right)=\left(\begin{array}{ccc}
1 & 0 & 0 \\
0 & \cos \theta & \sin \theta \\
0 & -\sin \theta & \cos \theta
\end{array}\right)\left(\begin{array}{c}
v^{i} \\
1 \\
n^{i} \\
1 \\
n^{i} \\
2
\end{array}\right)
$$

\section{The Expression of Special Curves in Weyl Space}

Definition 1. Let $C$ be a be a curve in three dimensional Weyl space. $C$ is called a general helix if the tangent vector field $v$ of $\mathrm{C}$ has constant angle $\varphi$ with some fixed vector field $u$, i.e.,

$$
g_{i j} v_{1}^{i} u^{j}=\cos \varphi=\text { constant }
$$

where $g_{i j} u^{i} u^{j}=1$.

Using Şemin (1983), we can express the condition to be a general helix in the following form:

Theorem 1. C is a general helix if and only if

$$
\frac{\kappa_{2}}{\kappa_{1}}=\text { constant }
$$

where $\kappa_{1}$ and $\kappa_{2}$ are the first and second curvatures of $C$.

With the help of Izumiya and Takeuchi (2004) and Kula et al. (2010) and using prolonged covariant derivative, the following proposition can be given:

Proposition 1. If C is a slant helix,

$$
\frac{\kappa_{1}^{2}}{\left(\kappa_{1}^{2}+\kappa_{2}^{2}\right)^{3 / 2}}\left(v_{1}^{k} \dot{\nabla}_{k} \frac{\kappa_{2}}{\kappa_{1}}\right)=\text { constant }
$$

is satisfied.

Proof. Let $C$ be a slant helix. Then $C$ is a curve with $\kappa_{1} \neq 0$ and $\left(v_{1}^{k} \dot{\nabla}_{k} \frac{\kappa_{2}}{\kappa_{1}}\right)$ is a constant function.

Let $\bar{C}: y^{i}=y^{i}(\bar{s})$ be the spherical image of principal normal vector field $v_{2}$ of $C(\bar{S}$ is the arc length parameter of $\bar{C})$. Then, using Frenet frame the following equalities are satisfied:

$$
\begin{aligned}
& v_{1}^{v^{k}} \dot{\nabla}_{k} y^{i}=v_{1}^{k} \dot{\nabla}_{k} v_{2}^{i} \\
& \left(\bar{v}_{1}^{k} \dot{\bar{\nabla}}_{k} y^{i}\right) a=-\kappa_{1} v_{1}^{i}+\kappa_{2}{\underset{3}{v}}^{i} \\
& \bar{v}_{1}^{i}=-\frac{\kappa_{1}}{\sqrt{\kappa_{1}^{2}+\kappa_{2}^{2}}} v_{1}^{i}+\frac{\kappa_{2}}{\sqrt{\kappa_{1}^{2}+\kappa_{2}^{2}}} \underbrace{i}
\end{aligned}
$$


where $\bar{v}$ is the tangent vector field of $\bar{C}$ and $a=a(s)=$ $\sqrt{\kappa_{1}^{2}+\kappa_{2}^{2}}$.

The prolonged covariant derivative of $\bar{v}^{i}$ in the direction of $v$ is

$$
\begin{aligned}
& \boldsymbol{v}_{1}^{k} \dot{\nabla}_{k} \bar{v}_{1}^{i}=\left(\bar{v}^{k} \dot{\bar{\nabla}}_{k} \bar{v}_{1}^{i}\right) a \\
& \bar{v}_{1}^{k} \dot{\bar{\nabla}}_{k} \bar{v}_{1}^{i}=\bar{\kappa}_{1} \bar{v}_{2}^{i}=\bar{c}_{1}^{i} \\
& =\frac{\kappa_{2} \kappa_{1}^{2}}{\left(\kappa_{1}^{2}+\kappa_{2}^{2}\right)^{2}}\left(v_{1}^{k} \dot{\nabla}_{k} \frac{\kappa_{2}}{\kappa_{1}}\right) v_{1}^{i} \\
& +\frac{\kappa_{1}^{3}}{\left(\kappa_{1}^{2}+\kappa_{2}^{2}\right)^{2}}\left(v_{1}^{k} \dot{\nabla}_{k} \frac{\kappa_{2}}{\kappa_{1}}\right) v_{3}^{i}-v_{2}^{i}
\end{aligned}
$$

where $\bar{\kappa}_{1}$ is the first curvature of $\bar{C}, \bar{v}$ is the principal normal vector field of $\bar{C}$ and $\bar{c}_{1}^{i}$ is the geodesic curvature vector field of the net $(\bar{v}, \bar{v}, \bar{v})$

The geodesic curvature of the spherical image of the principal normal vector field of $v$ is

$$
\kappa_{g}^{2}=g_{i j} \bar{c}_{1}^{i} \bar{c}^{j}=\bar{\kappa}_{1}^{2}=\left[v_{1}^{k} \dot{\nabla}_{k} \frac{\kappa_{2}}{\kappa_{1}}\right]^{2} \frac{\kappa_{1}^{4}}{\left(\kappa_{1}^{2}+\kappa_{2}^{2}\right)^{3}}+1
$$

Let us denote the first term of in the right hand side of the above equality by $\sigma^{2}$. Then, we get

$$
\bar{\kappa}_{1}^{2}=\sigma^{2}+1 .
$$

Since the spherical image of the principal normal vector field $\underset{2}{v}$ is a part of a circle in $S^{2}, \bar{\kappa}_{1}$ must be non-zero constant and so

$$
\sigma^{2}=\left[v_{1}^{k} \dot{\nabla}_{k} \frac{\kappa_{2}}{\kappa_{1}}\right]^{2} \frac{\kappa_{1}^{4}}{\left(\kappa_{1}^{2}+\kappa_{2}^{2}\right)^{3}}
$$

or

$$
\sigma=\left(v_{1}^{k} \dot{\nabla}_{k} \frac{\kappa_{2}}{\kappa_{1}}\right) \frac{\kappa_{1}^{2}}{\left(\kappa_{1}^{2}+\kappa_{2}^{2}\right)^{3 / 2}}
$$

is a constant function.

$$
\text { Here } \kappa_{1}=g_{i j}\left(\boldsymbol{v}_{1}^{k} \dot{\nabla}_{\boldsymbol{k}} \boldsymbol{v}_{1}^{i}\right) \boldsymbol{v}_{2}^{j}=\stackrel{2}{2}_{1}^{2} \text { and } \kappa_{1}=g_{i j}\left(\underset{1}{\boldsymbol{v}^{k}} \dot{\nabla}_{\boldsymbol{k}} \boldsymbol{v}^{i}\right) \underset{2}{\boldsymbol{v}^{j}}=
$$$$
-\tau_{31}^{2} \text {. }
$$

Further, by means of Kofoğlu (2020), the following theorem is valid:

Theorem 2. Let $C$ be a curve which has non-zero Bishop curvatures in $W_{3}$. $C$ is a slant helix if and only if $\frac{k_{1}}{k_{2}}$ is constant.

Using Şemin (1983), we can write the following proposition:

Proposition 2. If $\mathrm{C}$ is a spherical curve,

$$
\frac{\kappa_{2}}{\kappa_{1}}+v_{1}^{l} \dot{\nabla}_{l}\left[\frac{1}{\kappa_{2}}\left(v_{1}^{k} \dot{\nabla}_{k} \frac{1}{\kappa_{1}}\right)\right]=0 \quad(l=1,2,3)
$$

is satisfied.
Proof. Let $C$ be a spherical curve. If we choose center of the sphere as origin, the position vector at any point of $C$ satisfies the following relation:

$$
\left[g_{i j} x^{i} x^{j}\right]^{2}=R^{2}
$$

Here $R$ is the radius of the sphere and it is constant.

Taking prolonged covariant derivative of $g_{i j} x^{i} x^{j}=R$ in the direction of $v$, we get

$$
g_{i j} v_{1}^{i} x^{j}=0
$$

where $v$ is the tangent vector field of $C$.

Taking prolonged covariant derivative of (14) in the direction of $\underset{1}{v}$, we have

$$
\begin{gathered}
g_{i j}\left(\underset{1}{v^{k}} \dot{\nabla}_{k} v_{1}^{i}\right) x^{j}+1=0 \\
g_{i j} \kappa_{1} v_{2}^{i} x^{j}+1=0 \\
g_{i j} v_{2}^{i} x^{j}=-\frac{1}{\kappa_{1}}
\end{gathered}
$$

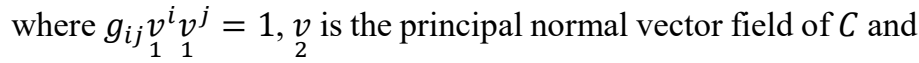
$\kappa_{1}$ is the first curvature of $C$.

Taking prolonged covariant derivative of (15) in the direction of $\underset{1}{v}$, we obtain

$$
\begin{aligned}
& -\kappa_{2} g_{i j} v_{3}^{i} x^{j}=v_{1}^{k} \dot{\nabla}_{k} \frac{1}{\kappa_{1}} \\
& -g_{i j} v_{3}^{i} x^{j}=\frac{1}{\kappa_{2}} v_{1}^{k} \dot{\nabla}_{k} \frac{1}{\kappa_{1}}
\end{aligned}
$$

where $v_{3}$ is the binormal vector field of $C, \kappa_{2}$ is the second curvature of $C, g_{i j} v_{1}^{i} v^{j}=0$ and $g_{i j} v_{1}^{i} x^{j}=0$.

Again taking prolonged covariant derivative of (16) in the direction of $\underset{\mathbf{1}}{ }$, we have

$$
\kappa_{2} g_{i j} v_{2}^{i} x^{j}=v_{1}^{l} \dot{\nabla}_{l}\left(\frac{1}{\kappa_{2}} v_{1}^{k} \dot{\nabla}_{k} \frac{1}{\kappa_{1}}\right)
$$

where $g_{i j} v_{3}^{i} v^{j}=0$.

Using (15) in (17), we get

$$
\frac{\kappa_{2}}{\kappa_{1}}+v_{1}^{l} \dot{\nabla}_{l}\left(\frac{1}{\kappa_{2}} v^{k} \dot{\nabla}_{k} \frac{1}{\kappa_{1}}\right)=0
$$

which concludes the proof. $\square$

With the help of Nomizu and Yano (1974) and Özdeğer and Şentürk (2002), the following definition and proposition can be formulated:

Definition 2. $C$ is called a circle if there exists a vector field $z^{i}$ and a positive constant $k$ such that 
where $g_{i j} z^{i} z^{j}=1$.

$$
\begin{gathered}
v_{1}^{k} \dot{\nabla}_{k} v_{1}^{i}=k z^{i} \\
v_{1}^{k} \dot{\nabla}_{k} z^{i}=-k v_{1}^{i}
\end{gathered}
$$

Proposition 3. If $C$ is a circle, the equation

$$
v_{1}^{l} \dot{\nabla}_{l}\left(v_{1}^{k} \dot{\nabla}_{k} v_{1}^{i}\right)+g_{i j}\left(v_{1}^{k} \dot{\nabla}_{k} v_{1}^{i}\right)\left(v_{1}^{k} \dot{\nabla}_{k} v_{1}^{j}\right) v_{1}^{i}=0
$$

is satisfied. Conversely, if $C$ satisfies the above equation, $C$ is either a geodesic or a circle.

\section{About The Spherical Images of a Curve in Weyl Space}

Definition 3. Let $C$ be a curve in $W_{3}$. If we translate the first vector field of type-1 Bishop frame to the center $O$ of the unit sphere $S^{2}$, we obtain a spherical image $\bar{C}: u^{i}=u^{i}(\bar{S})(\bar{S}$ is the arc length parameter of $\bar{C}) \cdot \bar{C}$ is called tangent Bishop spherical image or indicatrix of the curve $\bar{C}$.

In order to investigate the relations between type-1 Bishop and Frenet-Serret invariants we take the prolonged covariant derivative of $u^{i}$ in the direction of $\underset{1}{v}$, we have

$$
\begin{aligned}
v_{1}^{k} \dot{\nabla}_{k} u^{i} & =k_{1} \underline{1}^{i}+k_{2}{\underset{n}{n^{i}}}^{i} \\
\left(\bar{v}^{k} \dot{\bar{\nabla}}_{k} u^{i}\right) a & =k_{1} \boldsymbol{n}^{i}+k_{2} \underline{2}^{i} \\
\bar{v}^{i} a & =k_{1} \boldsymbol{n}_{1}^{i}+k_{2} \underline{n}^{i}
\end{aligned}
$$

where $\bar{v}_{1}^{i}$ is the tangent vector field of $\bar{C}, g_{i j} \bar{v}_{1}^{i} \bar{v}_{1}^{j}=1$ and $a=$ $a(s)$.

Taking the norm of both sides of (21), we get

$$
a=\mp \sqrt{k_{1}^{2}+k_{2}^{2}} \text {. }
$$

Let us take $a=\sqrt{k_{1}^{2}+k_{2}^{2}}$. Hence, we obtain

$$
\bar{v}_{1}^{i}=\frac{k_{1}}{\sqrt{k_{1}^{2}+k_{2}^{2}}} \stackrel{n}{i}^{i}+\frac{k_{2}}{\sqrt{k_{1}^{2}+k_{2}^{2}}} n^{i}
$$

where $k_{1}=g_{i j} c_{1}^{i} n^{j}$ and $k_{2}=g_{i j} c_{2}^{i} n_{2}^{j}$.

Taking the prolonged covariant derivative of (23) in the direction of $v$, we have

$$
\begin{aligned}
& \boldsymbol{v}_{1}^{k} \dot{\nabla}_{k} \bar{v}_{1}^{i}=\left(\bar{v}^{k} \dot{\bar{\nabla}}_{k} \bar{v}^{i}\right) a \\
& =\frac{k_{2}^{3}}{\left(k_{1}^{2}+k_{2}^{2}\right)^{3 / 2}}\left(v_{1}^{k} \dot{\nabla}_{k} \frac{k_{1}}{k_{2}}\right){ }_{1}^{n^{i}} \\
& +\frac{\boldsymbol{k}_{1}^{3}}{\left(\boldsymbol{k}_{1}^{2}+\boldsymbol{k}_{2}^{2}\right)^{3 / 2}}\left(v_{1}^{k} \dot{\nabla}_{k} \frac{\boldsymbol{k}_{2}}{\boldsymbol{k}_{1}}\right) n_{2}^{i} \\
& -\sqrt{k_{1}^{2}+k_{2}^{2}} v_{1}^{i}
\end{aligned}
$$

and

$$
\begin{aligned}
\overline{\boldsymbol{\kappa}}_{1} \bar{v}^{i}= & \frac{k_{2}^{3}}{\left(k_{1}^{2}+k_{2}^{2}\right)^{2}}\left(\underset{1}{v^{k}} \dot{\nabla}_{k} \frac{k_{1}}{k_{2}}\right) \underset{1}{n_{1}^{i}} \\
& +\frac{k_{1}^{3}}{\left(k_{1}^{2}+k_{2}^{2}\right)^{2}}\left(v_{1}^{k} \dot{\nabla}_{k} \frac{k_{2}}{k_{1}}\right) \underset{2}{n^{i}-v_{1}^{i}}
\end{aligned}
$$

where $k_{1}=g_{i j} c_{1}^{i} n^{j}$ and $k_{2}=g_{i j} c_{2}^{i} n^{j}$.

Taking the norm of both sides of (25), we get

$$
\begin{aligned}
\bar{\kappa}_{1}=\left(\left[\frac{k_{2}^{3}}{\left(k_{1}^{2}+k_{2}^{2}\right)^{2}}\left(v_{1}^{k} \dot{\nabla}_{k} \frac{k_{1}}{k_{2}}\right)\right]^{2}\right. \\
\left.+\left[\frac{k_{1}^{3}}{\left(k_{1}^{2}+k_{2}^{2}\right)^{2}}\left(v_{1}^{k} \dot{\nabla}_{k} \frac{k_{2}}{k_{1}}\right)\right]^{2}+1\right)^{1 / 2}
\end{aligned}
$$

and from (24) and (26)

$$
\begin{aligned}
\underset{2}{\bar{v}^{i}}=\frac{1}{\bar{\kappa}_{1}} & \frac{k_{2}^{3}}{\left(k_{1}^{2}+k_{2}^{2}\right)^{2}}\left(v_{1}^{k} \dot{\nabla}_{k} \frac{k_{1}}{k_{2}}\right)_{1}^{n_{1}^{i}} \\
& +\frac{1}{\bar{\kappa}_{1}} \frac{k_{1}^{3}}{\left(k_{1}^{2}+k_{2}^{2}\right)^{2}}\left(v_{1}^{k} \dot{\nabla}_{k} \frac{k_{2}}{k_{1}}\right)_{2}^{n^{i}}-\frac{1}{\bar{\kappa}_{1}} v^{i}
\end{aligned}
$$

where $\bar{\kappa}_{1}$ is the first curvature of $\bar{C}, \bar{v}_{2}^{i}$ is the principal vector field of $\bar{C}, g_{i j} \bar{v}_{2}^{i} \bar{v}^{j}=1, k_{1}=g_{i j} c_{1}^{i} n_{1}^{j}$ and $k_{2}=g_{i j} c_{2}^{i} n_{2}^{j}$.

We know that

$$
\bar{v}^{i}=\epsilon_{i j k} \bar{v}_{1}^{j} \bar{v}^{k}
$$

Using (23) and (27) in (28), we obtain

$$
\begin{array}{r}
\bar{v}_{3}^{i}=\frac{1}{\bar{\kappa}_{1}}\left\{\left[\frac{k_{1}^{4}}{\left(k_{1}^{2}+k_{2}^{2}\right)^{5 / 2}}\left(v_{1}^{k} \dot{\nabla}_{k} \frac{k_{2}}{k_{1}}\right)\right.\right. \\
\left.-\frac{k_{2}^{4}}{\left(k_{1}^{2}+k_{2}^{2}\right)^{5 / 2}}\left(v_{1}^{k} \dot{\nabla}_{k} \frac{k_{1}}{k_{2}}\right)\right] v_{1}^{i} \\
\left.-\frac{k_{2}}{\sqrt{k_{1}^{2}+k_{2}^{2}}} n_{1}^{i}+\frac{k_{1}}{\sqrt{k_{1}^{2}+k_{2}^{2}}} n^{i}\right\}
\end{array}
$$

where $k_{1}=g_{i j} c_{1}^{i} n_{1}^{j}$ and $k_{2}=g_{i j} c_{2}^{i} n^{j}$.

Taking the prolonged covariant derivative of (29) in the direction of $\underset{1}{v}$ and multiplying this expression by $g_{i j} \bar{v}_{2}^{j}$, we get

$$
\begin{aligned}
& \bar{\kappa}_{2}=\frac{1}{\left(k_{1}^{2}+k_{2}^{2}\right)^{3}+\left[k_{1}^{2} v^{k} \dot{\nabla}_{k} \frac{k_{2}}{k_{1}}\right]^{2}} \\
& \left\{\left[v_{1}^{l} \dot{\nabla}_{l}\left(v_{1}^{k} \dot{\nabla}_{k} k_{2}\right)\right] k_{1}\left(k_{1}^{2}+k_{2}^{2}\right)\right. \\
& -\left[v_{1}^{l} \dot{\nabla}_{l}\left(v_{1}^{k} \dot{\nabla}_{k} k_{1}\right)\right] k_{2}\left(k_{1}^{2}+k_{2}^{2}\right) \\
& -3 k_{1}^{2}\left(v_{1}^{k} \dot{\nabla}_{k} k_{1}\right)\left(v_{1}^{k} \dot{\nabla}_{k} k_{2}\right) \\
& +3 k_{1} k_{2}\left(v_{1}^{k} \dot{\nabla}_{k} k_{1}\right)\left(v_{1}^{k} \dot{\nabla}_{k} k_{1}\right) \\
& -3 k_{1} k_{2}\left(v_{1}^{k} \dot{\nabla}_{k} k_{2}\right)\left(v_{1}^{k} \dot{\nabla}_{k} k_{2}\right)
\end{aligned}
$$




$$
\left.+3 k_{2}^{2}\left(v_{1}^{k} \dot{\nabla}_{k} k_{1}\right)\left(v_{1}^{k} \dot{\nabla}_{k} k_{2}\right)\right\}
$$

where $\bar{\kappa}_{2}$ is the second curvature of $\bar{C}$ and $g_{i j} \bar{v}_{2}^{i} \bar{v}_{2}^{j}=1, k_{1}=$ $g_{i j} c_{1}^{i} n^{j}$ and $k_{2}=g_{i j} c_{1}^{i} n^{j}$

Corollary 1. Let $\bar{C}$ be the tangent Bishop spherical image of $C$. If $\frac{k_{1}}{k_{2}}=$ constant, then the tangent Bishop spherical image $u^{i}=$ $u^{i}(\bar{s})$ is a circle.

Proof. If we use Proposition 3, we have

$$
\begin{aligned}
& {\underset{1}{v}}^{l} \dot{\nabla}_{l}\left(\underline{v}_{1}^{k} \dot{\nabla}_{k} \bar{v}_{1}^{i}\right) \\
& =\left[v_{1}^{l} \dot{\nabla}_{l} \frac{k_{2}^{3}}{\left(k_{1}^{2}+k_{2}^{2}\right)^{3 / 2}}\right]\left(v_{1}^{k} \dot{\nabla}_{k} \frac{k_{1}}{k_{2}}\right){ }_{1}^{n^{i}} \\
& +\frac{k_{2}^{3}}{\left(k_{1}^{2}+k_{2}^{2}\right)^{\frac{3}{2}}}\left[\underset{1}{v^{l}} \dot{\nabla}_{l}\left(\underset{1}{v^{k}} \dot{\nabla}_{k} \frac{k_{1}}{k_{2}}\right)\right]_{1}^{n^{i}} \\
& -\left[v_{1}^{l} \dot{\nabla}_{l} \frac{k_{1} k_{2}^{2}}{\left(k_{1}^{2}+k_{2}^{2}\right)^{\frac{3}{2}}}\right]\left(v_{1}^{k} \dot{\nabla}_{k} \frac{k_{1}}{k_{2}}\right){\underset{2}{n}}^{i} \\
& -\frac{k_{1} k_{2}^{2}}{\left(k_{1}^{2}+k_{2}^{2}\right)^{3 / 2}} v_{1}^{l} \dot{\nabla}_{l}\left(v_{1}^{k} \dot{\nabla}_{k} \frac{k_{1}}{k_{2}}\right) n_{2}^{i} \\
& -\frac{1}{\sqrt{k_{1}^{2}+k_{2}^{2}}} \\
& \left\{k_{2}\left(\underset{1}{\left.v_{l}^{l} \dot{\nabla}_{l} k_{2}\right)} \frac{\left(k_{1}^{2}+k_{2}^{2}\right)}{k_{2}^{2}}+k_{2} k_{1}\left(\underset{1}{v^{l}} \dot{\nabla}_{l} \frac{k_{1}}{k_{2}}\right)\right\}{\underset{1}{i}}^{i}\right. \\
& -\sqrt{k_{1}^{2}+k_{2}^{2}}\left(k_{1} \boldsymbol{n}^{i}+k_{2} \underset{2}{n^{i}}\right)
\end{aligned}
$$

and

$$
\begin{aligned}
g_{i j} & \left(\underset{1}{v^{k}} \dot{\nabla}_{k} \bar{v}_{1}^{i}\right)\left(\underset{1}{v^{k}} \dot{\nabla}_{k} \bar{v}_{1}^{j}\right) \underset{1}{\bar{v}^{i}} \\
& =\left\{k_{2}^{4} \frac{1}{\left(k_{1}^{2}+k_{2}^{2}\right)^{2}}\left[\underset{1}{v^{k}} \dot{\nabla}_{k} \frac{k_{1}}{k_{2}}\right]^{2}+\left(k_{1}^{2}+k_{2}^{2}\right)\right\} \bar{v}^{i}
\end{aligned}
$$

where $k_{1}=g_{i j} c_{1}^{i} n^{j}$ and $k_{2}=g_{i j} c_{2}^{i} n_{2}^{j}$.

Under the condition $\frac{k_{1}}{k_{2}}=$ constant, using (31) and (32), we obtain

$$
\underset{1}{v^{l}} \dot{\nabla}_{l}\left(\underset{1}{v^{k}} \dot{\nabla}_{k} \bar{v}_{1}^{i}\right)+g_{i j}\left(\underset{1}{v^{k}} \dot{\nabla}_{k} \bar{v}_{1}^{i}\right)\left(v_{1}^{k} \dot{\nabla}_{k} \bar{v}_{1}^{j}\right) \bar{v}_{1}^{i}=0
$$

Hence, we see that, the tangent Bishop spherical image $u^{i}=$ $u^{i}(\bar{s})$ of $C$ is a circle.

Definition 4. Let $C$ be a curve in $W_{3}$. If we translate the second vector field of type-1 Bishop frame to the center $O$ of the unit sphere $S^{2}$, we obtain a spherical image $\bar{F}: f^{i}=f^{i}(\bar{s})(\bar{s}$ is the arc length parameter of $\bar{F}$ ). $\bar{F}$ is called $n$ Bishop spherical image or indicatrix of the curve $\mathrm{C}$.
The relations between type-1 Bishop and Frenet-Serret invariants are obtained by taking the prolonged covariant derivative of $f^{i}$ in the direction of $v$, we have

$$
\begin{aligned}
{\underset{1}{v}}^{k} \dot{\nabla}_{k} f^{i} & =-k_{1}{\underset{1}{v}}^{i} \\
\left(\bar{v}^{k} \dot{\bar{\nabla}}_{k} f^{i}\right) b & =-k_{1}{\underset{1}{v^{i}}}^{i} \\
\bar{v}^{i} b & =-k_{1}{\underset{1}{v}}^{i}
\end{aligned}
$$

where $\bar{v}_{1}^{i}$ is the tangent vector field of $\bar{F}, g_{i j} \bar{v}_{1}^{i} \bar{v}_{1}^{j}=1$ and $b=$ $b(s)$.

Taking the norm of both sides of (36), we get

$$
\boldsymbol{b}=\mp \boldsymbol{k}_{1}
$$

Let us take $b=-k_{1}$. In this case, we obtain

$$
\bar{v}^{i}=v_{1}^{i}
$$

Taking the prolonged covariant derivative of (38) in the direction of $v$, we have

$$
\begin{aligned}
& v_{1}^{k} \dot{\nabla}_{k} \bar{v}^{i}=\left(\bar{v}_{1}^{k} \dot{\bar{\nabla}}_{k} \bar{v}_{1}^{i}\right) b=v_{1}^{k} \dot{\nabla}_{k} v_{1}^{i} \\
& \bar{M}_{1} \bar{v}^{i}\left(-k_{1}\right)=k_{1} \underset{1}{n^{i}}+k_{2} \underset{2}{n^{i}} \\
& \bar{M}_{1} \bar{v}^{i}=-\underset{1}{n^{i}}-\frac{k_{2}}{k_{1}} \boldsymbol{n}^{i}
\end{aligned}
$$

Taking the norm of both sides of (41), we get

$$
\bar{M}_{1}=\sqrt{1+\left(\frac{k_{2}}{k_{1}}\right)^{2}}=\sqrt{1+\left(\frac{g_{i j} c_{1}^{i} n^{j}}{g_{i j} c_{1}^{i} n_{1}^{j}}\right)^{2}}
$$

and

$$
\bar{v}_{2}^{i}=-\frac{1}{\bar{M}_{1}}{\underset{1}{i}}^{i}-\frac{1}{\bar{M}_{1}} \frac{k_{2}}{k_{1}} \underset{2}{n^{i}}
$$

where $\bar{v}_{2}^{i}$ is the principal normal vector field of $\bar{F}, g_{i j} \bar{v}_{2}^{i} \bar{v}_{2}^{j}=1$ and $\bar{M}_{1}$ is the first curvature of $\bar{F}$.

$\bar{v}^{i}$ is the binormal vector field of $\bar{F}$ and it is defined in the form:

$$
\bar{v}^{i}=\epsilon_{i j k} \bar{v}_{1}^{j} \bar{v}^{k}
$$

If (38) and (43) are used in (44), we have

$$
\underset{3}{\bar{v}^{i}}=-\frac{1}{\bar{M}_{1}} \underset{2}{n^{i}}+\frac{1}{\bar{M}_{1}} \frac{k_{2}}{k_{1}} \underset{1}{n^{i}}
$$

Taking the prolonged covariant derivative of (45) in the direction of $v$, we get 


$$
\begin{aligned}
\underset{1}{v^{k}} \dot{\nabla}_{k} \bar{v}^{i}=\left(\underset{1}{\bar{v}^{k}} \dot{\bar{\nabla}}_{k}\right. & \left.\bar{v}^{i}\right) b=-\bar{M}_{2} \bar{v}_{2}^{i}\left(-k_{1}\right) \\
= & -\left(\underset{1}{v^{k}} \dot{\nabla}_{k} \frac{1}{\bar{M}_{1}}\right) \underset{2}{n^{i}} \\
& +\left(\underset{1}{v^{k}} \dot{\nabla}_{k} \frac{1}{\bar{M}_{1}}\right) \frac{k_{2}}{k_{1}}{\underset{1}{i}}^{i} \\
& +\frac{1}{\bar{M}_{1}}\left(\underset{1}{v^{k}} \dot{\nabla}_{k} \frac{k_{2}}{k_{1}}\right) \underset{1}{n^{i}}
\end{aligned}
$$

and multiplying (46) by $g_{i j} \bar{v}_{2}^{j}$, we obtain

$$
\bar{M}_{2}=-\frac{1}{\left[\bar{M}_{1}\right]^{2}} \frac{1}{k_{1}}\left(v_{1}^{k} \dot{\nabla}_{k} \frac{k_{2}}{k_{1}}\right)=-\frac{k_{1}}{k_{1}^{2}+k_{2}^{2}} v_{1}^{k} \dot{\nabla}_{k} \frac{k_{2}}{k_{1}}
$$

or

$$
\bar{M}_{2}=-\frac{g_{i j} c_{1}^{i} n_{1}^{j}}{\left(g_{i j} c_{1}^{i} n^{j}\right)^{2}+\left(g_{i j} c_{2}^{i} n^{j}\right)^{2}} v_{1}^{k} \dot{\nabla}_{k}\left[\frac{g_{i j} c_{1}^{i} n_{2}^{j}}{g_{i j} c_{1}^{i} n^{j}}\right]
$$

where $\bar{M}_{2}$ is the second curvature of $\bar{F}$.

Corollary 2. Let $\bar{F}$ be $n_{1}$ Bishop spherical image of $C$. If $\frac{k_{1}}{k_{2}}=$ constant, then $\underset{1}{n}$ Bishop spherical image $f^{i}=f^{i}(\bar{s})$ is a circle.

Proof. By using Proposition 3, we get

$$
\begin{aligned}
& v_{1}^{l} \dot{\nabla}_{l}\left(\underset{1}{v^{k}} \dot{\nabla}_{k} \bar{v}_{1}^{i}\right)=v_{1}^{l} \dot{\nabla}_{l}\left(v_{1}^{k} \dot{\nabla}_{k} v_{1}^{i}\right) \\
& =\left(v_{1}^{l} \dot{\nabla}_{l} k_{1}\right) \underset{1}{n^{i}}+\left(\underset{1}{v^{l}} \dot{\nabla}_{l} k_{2}\right) \underset{2}{n^{i}} \\
& -\left(\boldsymbol{k}_{1}^{2}+\boldsymbol{k}_{2}^{2}\right) v_{1}^{i}
\end{aligned}
$$

and

$$
\begin{aligned}
g_{i j}\left(v_{1}^{k} \dot{\nabla}_{k} \bar{v}_{1}^{i}\right)\left(v_{1}^{k} \dot{\nabla}_{k} \bar{v}_{1}^{j}\right) \underset{1}{v^{i}} & =g_{i j}\left(v_{1}^{k} \dot{\nabla}_{k} v_{1}^{i}\right)\left(v_{1}^{k} \dot{\nabla}_{k} v_{1}^{j}\right) v_{1}^{i} \\
& =\left(k_{1}^{2}+k_{2}^{2}\right) v_{1}^{i}
\end{aligned}
$$

Summing (49) and (50), we have

$$
\begin{aligned}
\underset{1}{v^{l}} \dot{\nabla}_{l}\left(v_{1}^{v^{k}} \dot{\nabla}_{k} \bar{v}^{i}\right)+ & g_{i j}\left(\underset{1}{v^{k}} \dot{\nabla}_{k} \bar{v}^{i}\right)\left(\underset{1}{v^{k}} \dot{\nabla}_{k} \bar{v}^{j}\right) \bar{v}_{1}^{i} \\
& =\left(\underset{1}{\left.v^{l} \dot{\nabla}_{l} k_{1}\right)}\right)_{1}^{n_{1}^{i}}+\left(\underset{1}{v^{l} \dot{\nabla}_{l} k_{2}}\right) \underset{2}{n^{i}}
\end{aligned}
$$

Using $k_{1}=\kappa_{1} \cos \theta$ and $k_{2}=\kappa_{1} \sin \theta$, we obtain

$$
\begin{aligned}
& v_{1}^{l} \dot{\nabla}_{l} k_{1}=v_{1}^{l} \dot{\nabla}_{l}\left(\kappa_{1} \cos \theta\right) \\
& =\left(v_{1}^{l} \dot{\nabla}_{l} \kappa_{1}\right) \cos \theta-\kappa_{1}\left(v_{1}^{l} \dot{\nabla}_{l} \theta\right) \sin \theta
\end{aligned}
$$

and

$$
\begin{aligned}
& v_{1}^{l} \dot{\nabla}_{l} k_{2}=v_{1}^{l} \dot{\nabla}_{l}\left(\kappa_{1} \sin \theta\right) \\
& =\left(\underset{1}{\left.v^{l} \dot{\nabla}_{l} \kappa_{1}\right)}\right) \sin \theta+\kappa_{1}\left(\underset{1}{v^{l} \dot{\nabla}_{l} \theta}\right) \cos \theta
\end{aligned}
$$

where $\theta=\theta(s)=\operatorname{arccot} \frac{k_{1}}{k_{2}}$ and $v_{1}^{k} \dot{\nabla}_{k} \theta=\frac{v^{k} \dot{\nabla}_{k}}{1+\left(\frac{k_{1}}{k_{2}}\right)^{2}}$.

It is known that $\kappa_{1}=\sqrt{k_{1}^{2}+k_{2}^{2}}$. In this case, we obtain

$$
\begin{aligned}
\underset{1}{v^{k}} \dot{\nabla}_{k} \kappa_{1}= & \underset{1}{v^{k}} \dot{\nabla}_{k} \sqrt{k_{2}^{2}\left[\left(\frac{k_{1}}{k_{2}}\right)^{2}+1\right]} \\
= & \frac{1}{\sqrt{k_{1}^{2}+k_{2}^{2}}-k_{2} \sin \theta\left(\frac{k_{1}^{2}+k_{2}^{2}}{k_{2}^{2}}\right)} \\
& \cdot\left\{k_{2} \kappa_{1}\left(\underset{1}{v^{k}} \dot{\nabla}_{k} \theta\right) \cos \theta\left(\frac{k_{1}^{2}+k_{2}^{2}}{k_{2}^{2}}\right)\right. \\
& \left.+k_{2} k_{1}\left(\underset{1}{v^{k}} \dot{\nabla}_{k} \frac{k_{1}}{k_{2}}\right)\right\}
\end{aligned}
$$

If $\frac{k_{1}}{k_{2}}=$ constant, we have $v_{1}^{k} \dot{\nabla}_{k} \theta=0$ and $v_{1}^{k} \dot{\nabla}_{k} \kappa_{1}=0$. This means $v_{1}^{k} \dot{\nabla}_{k} k_{1}=0$ and $\underset{1}{v^{k}} \dot{\nabla}_{k} k_{2}=0$. Using these results in (51),

$$
\underset{1}{v^{l} \dot{\nabla}_{l}}\left(v_{1}^{k} \dot{\nabla}_{k} \bar{v}^{i}\right)+g_{i j}\left(v_{1}^{k} \dot{\nabla}_{k} \bar{v}_{1}^{i}\right)\left(\underset{1}{v^{k}} \dot{\nabla}_{k} \bar{v}_{1}^{j}\right) \bar{v}_{1}^{i}=0
$$

is obtained. So, it is shown that $\bar{F}: f^{i}=f^{i}(\bar{S})$ is a circle. $\square$

Theorem 3. Let $\bar{F}: f^{i}=f^{i}(\bar{s})$ be $n$ Bishop spherical image of $C$. If $\bar{F}$ is a general helix, then

$$
\frac{k_{1}^{2}}{\left(k_{1}^{2}+k_{2}^{2}\right)^{3 / 2}}\left(v_{1}^{k} \dot{\nabla}_{k} \frac{k_{2}}{k_{1}}\right)=\text { constant }
$$

is valid.

Theorem 4. Let $\bar{F}: f^{i}=f^{i}(\bar{s})$ be $n$ Bishop spherical image of $C$. If $\bar{F}$ is a slant helix, then

$$
\frac{\left(k_{1}^{2}+k_{2}^{2}\right)^{4} k_{1}}{\left\{\left(k_{1}^{2}+k_{2}^{2}\right)^{3}+k_{1}^{4}\left[v_{1}^{k} \dot{\nabla}_{k} \frac{k_{2}}{k_{1}}\right]^{2}\right\}^{3 / 2}} v_{1}^{l} \dot{\nabla}_{l}\left[\frac{k_{1}^{2} v_{1}^{k} \dot{\nabla}_{k} \frac{k_{2}}{k_{1}}}{\left(k_{1}^{2}+k_{2}^{2}\right)^{3 / 2}}\right]=\text { constant } \text {. }
$$

Since $\bar{F}: f^{i}=f^{i}(\bar{s})$ is a spherical curve, by using Proposition 2, we can state the following theorem:

Theorem 5. Let $\bar{F}: f^{i}=f^{i}(\bar{s})$ be $n_{1}$ Bishop spherical image of $C$. In this case, the following equation

$$
\frac{k_{1}^{2}}{\left(k_{1}^{2}+k_{2}^{2}\right)^{3 / 2}}\left(v_{1}^{k} \dot{\nabla}_{k} \frac{k_{2}}{k_{1}}\right)-v_{1}^{k} \dot{\nabla}_{k} \frac{k_{1} k_{2}}{\sqrt{k_{1}^{2}+k_{2}^{2}}}=\text { constant } \text {. }
$$

is valid.

Theorem 6. Let $\bar{F}: f^{i}=f^{i}(\bar{s})$ be $n_{1}$ Bishop spherical image of C. If $\frac{k_{2}}{k_{1}}=$ constant, then $\underset{3}{\bar{v}}$ is parallel translated along $\underset{1}{\bar{v}}$.

Proof. From (46), it is known that 


$$
\begin{aligned}
\underline{1}^{k} \dot{\nabla}_{k} \bar{v}^{i}=\left(\bar{v}_{1}^{k} \dot{\bar{\nabla}}_{k} \bar{v}_{3}^{i}\right) & \left(-k_{1}\right) \\
= & -\left(v_{1}^{k} \dot{\nabla}_{k} \frac{1}{\bar{M}_{1}}\right) \underset{2}{n^{i}} \\
& +\left(v^{k} \dot{\nabla}_{k} \frac{1}{\bar{M}_{1}}\right) \frac{k_{2}}{k_{1}} n^{i} \\
& +\frac{1}{\bar{M}_{1}}\left(v_{1}^{k} \dot{\nabla}_{k} \frac{k_{2}}{k_{1}}\right)_{1}^{n^{i}}
\end{aligned}
$$

and

$$
\begin{aligned}
& \overline{\boldsymbol{v}}^{k} \dot{\bar{\nabla}}_{k} \overline{\bar{v}}^{i}=\overline{\boldsymbol{a}}_{31}^{i}=\frac{1}{\boldsymbol{k}_{1}}\left(v_{1}^{k} \dot{\bar{\nabla}}_{k} \frac{1}{\overline{\boldsymbol{M}}_{1}}\right) \boldsymbol{n}_{2}^{i} \\
& -\frac{k_{2}}{k_{1}^{2}}\left(v_{1}^{k} \dot{\nabla}_{k} \frac{1}{\bar{M}_{1}}\right) \underset{1}{n^{i}} \\
& -\frac{1}{k_{1}} \frac{1}{\bar{M}_{1}}\left(v_{1}^{k} \dot{\nabla}_{k} \frac{k_{2}}{k_{1}}\right) n_{1}^{i}
\end{aligned}
$$

where $\underset{31}{\bar{a}^{i}}$ is defined $\underset{31}{\bar{a}^{i}}=\underset{31 p}{\bar{\tau}} \bar{v}^{i}={ }_{\bar{T}_{k}}^{p} \bar{v}_{1}^{k} \bar{v}_{p}^{i}$ and it is named the Chebyshev vector field of the first kind of the net $\left(\begin{array}{l}\bar{v}, \bar{v} \\ \left.\frac{1}{2}, \frac{v}{3}\right)\end{array}\right.$ Besides, ${ }_{31} \bar{\tau}=\bar{T}_{k} \bar{T}_{1} \bar{v}^{k}$ is called the Chebyshev curvature of the first kind of the net $(\bar{v}, \bar{v}, \bar{v})$ (Tsareva and Zlatanov, 1990).

By taking prolong covariant derivative of $\frac{1}{\bar{M}_{1}}$ in the direction of $v$, we get

$$
v_{1}^{k} \dot{\nabla}_{k} \frac{1}{\bar{M}_{1}}=-\frac{k_{1}^{2} k_{2}}{\left(k_{1}^{2}+k_{2}^{2}\right)^{3 / 2}}\left(v_{1}^{k} \dot{\nabla}_{k} \frac{k_{2}}{k_{1}}\right)
$$

By using (58) in (57) and by considering the condition $\frac{k_{2}}{k_{1}}=$ constant, we have

$$
\underset{31}{\bar{a}^{i}}=0
$$

It means that $\underset{3}{\bar{v}}$ is parallel translated along $\underset{1}{\bar{v}}$. $\square$

Definition 5. Let $C$ be a curve in $W_{3}$. If we translate the third vector field of type-1 Bishop frame to the center $O$ of the unit sphere $S^{2}$, we obtain $\bar{G}: g^{i}=g^{i}(\bar{S})$ ( $\bar{s}$ is the arc length parameter of $\bar{G}), \bar{G}$ is called $\eta_{2}^{i}$ Bishop spherical image or indicatrix of the curve $C$.

Let us express the relations between type-1 Bishop and Frenet-Serret invariants similarly by taking prolonged covariant derivative of $g^{i}$ in the direction of $v_{1}$, we get

$$
\begin{gathered}
v_{1}^{k} \dot{\nabla}_{k} g^{i}=-k_{2} v_{1}^{i} \\
\left(\bar{v}^{k} \dot{\bar{\nabla}}_{k} g^{i}\right) c=-k_{2} v_{1}^{i} \\
\bar{v}^{i} c=-k_{2} v_{1}^{i}
\end{gathered}
$$

where $\bar{v}_{1}^{i}$ is the tangent vector field of $\bar{G}, g_{i j} \bar{v}_{1}^{i} \bar{v}_{1}^{j}=1$ and $c=$ $c(s)$.
Taking the norm of both sides of (62), we have

$$
\boldsymbol{c}=\mp \boldsymbol{k}_{2} .
$$

Let us choose $c=-k_{2}$. Then, we obtain

$$
\bar{v}^{i}=v_{1}^{i}
$$

By taking prolonged covariant derivative of (64) in the direction of $v$, we have

$$
\begin{aligned}
v_{1}^{k} \dot{\nabla}_{k} \bar{v}_{1}^{i} & =\left(\bar{v}_{1}^{k} \dot{\nabla}_{k} \bar{v}_{1}^{i}\right) c=v_{1}^{k} \dot{\nabla}_{k} v_{1}^{i} \\
\bar{H}_{1} \bar{v}_{2}^{i}\left(-k_{2}\right) & =k_{1}{ }_{1}^{i}+k_{2} n_{2}^{i} \\
\bar{H}_{1} \bar{v}^{i} & =-\frac{k_{1}}{k_{2}} \underline{n}_{1}^{i}-n_{2}^{i} .
\end{aligned}
$$

By taking the norm of both sides of (67), we get

$$
\bar{H}_{1}=\sqrt{\left(\frac{k_{1}}{k_{2}}\right)^{2}+1}=\sqrt{\left[\frac{g_{i j} c_{2}^{i} n_{2}^{j}}{g_{i j} c_{1}^{i} n_{1}^{j}}\right]^{2}+1}
$$

and

$$
\bar{v}^{i}=-\frac{1}{\bar{H}_{1}} \frac{k_{1}}{k_{2}} n^{i}-\frac{1}{\bar{H}_{1}} n^{i}
$$

where $\bar{v}_{2}^{i}$ is the principal normal vector field of $\bar{G}, g_{i j} \bar{v}_{2}^{i} \bar{v}_{2}^{j}=1$ and $\bar{H}_{1}$ is the first curvature of $\bar{G}$.

$\bar{v}_{3}^{i}$ is the binormal vector field of $\bar{G}$ and it is expressed as follows:

$$
\bar{v}^{i}=\epsilon_{i j k} \bar{v}_{1}^{j} \bar{v}^{k}
$$

By using (64) and (69) in (70), we have

$$
\bar{v}^{i}=-\frac{1}{\bar{H}_{1}} \frac{k_{1}}{k_{2}} n^{i}-\frac{1}{\bar{H}_{1}} n^{i}
$$

Taking the prolonged covariant derivative of (71) in the direction of $v$, we obtain

$$
\begin{aligned}
& v_{1}^{k} \dot{\nabla}_{k} \bar{v}_{3}^{i}=\left(\bar{v}_{1}^{k} \dot{\bar{\nabla}}_{k} \bar{v}_{3}^{i}\right) c=-\bar{H}_{2} \bar{v}_{2}^{i}\left(-k_{2}\right) \\
& =-\left(v_{1}^{k} \dot{\nabla}_{k} \frac{1}{\bar{H}_{1}}\right) \frac{k_{1}}{k_{2}} n^{i} \\
& -\frac{1}{\bar{H}_{1}}\left(v_{1}^{k} \dot{\nabla}_{k} \frac{k_{1}}{k_{2}}\right){\underset{2}{n}}^{i} \\
& +\left(\boldsymbol{v}^{k} \dot{\nabla}_{\boldsymbol{k}} \frac{1}{\overline{\boldsymbol{H}}_{1}}\right) \boldsymbol{n}_{1}^{i}
\end{aligned}
$$

and then multiplying (72) by $g_{i j} \bar{\nu}_{2}^{j}$, we get 


$$
\bar{H}_{2}=\left(\frac{1}{\bar{H}_{1}}\right)^{2} \frac{1}{k_{2}}\left(v_{1}^{k} \dot{\nabla}_{k} \frac{k_{1}}{k_{2}}\right)=\frac{k_{2}}{k_{1}^{2}+k_{2}^{2}}\left(v_{1}^{k} \dot{\nabla}_{k} \frac{k_{1}}{k_{2}}\right)
$$

or

$$
\bar{H}_{2}=\frac{g_{i j} c_{2}^{i} n_{2}^{j}}{\left(g_{i j} c_{1}^{i} n^{j}\right)^{2}+\left(g_{i j} c_{2}^{i} n_{2}^{j}\right)^{2}} v^{k} \dot{\nabla}_{k}\left[\frac{g_{i j} c_{1}^{i} n^{j}}{g_{i j} c_{2}^{i} n_{2}^{j}}\right]
$$

where $\bar{H}_{2}$ is the second curvature of $\bar{G}$.

Corollary 3. Let $\bar{G}$ be $\underset{2}{n}$ Bishop spherical image of $C$. If $\frac{k_{1}}{\boldsymbol{k}_{2}}=$ constant, then $\underset{2}{n}$ Bishop spherical image $g^{i}=g^{i}(\bar{s})$ is a circle.

Proof. It is the same with the proof of Corollary 2. $\square$

Theorem 7. Let $\bar{G}: g^{i}=g^{i}(\bar{s})$ be $\underset{2}{n}$ Bishop spherical image of $C$. If $\bar{G}$ is a general helix, then

$$
\frac{k_{2}^{2}}{\left(k_{1}^{2}+k_{2}^{2}\right)^{3 / 2}}\left(v_{1}^{k} \dot{\nabla}_{k} \frac{k_{1}}{k_{2}}\right)=\text { constant }
$$

is satisfied.

Theorem 8. Let $\bar{G}: g^{i}=g^{i}(\bar{s})$ be $\underset{2}{n}$ Bishop spherical image of $C$. If $\bar{G}$ is a slant helix, then

$$
\begin{gathered}
\frac{k_{2}\left(k_{1}^{2}+k_{2}^{2}\right)^{4}}{\left\{\left(k_{1}^{2}+k_{2}^{2}\right)^{3}+k_{1}^{4}\left[v^{k} \dot{\nabla}_{k} \frac{k_{1}}{k_{2}}\right]^{2}\right\}^{3 / 2}} v_{1}^{l} \dot{\nabla}_{l}\left[\frac{k_{2}^{2}}{\left(k_{1}^{2}+k_{2}^{2}\right)^{3 / 2}} v_{1}^{k} \dot{\nabla}_{k} \frac{k_{1}}{k_{2}}\right] \\
=\text { constant }
\end{gathered}
$$

is valid.

Since $\bar{G}: g^{i}=g^{i}(\bar{s})$ is spherical curve, with the help of Proposition 2, we can state the following theorem:

Theorem 9. Let $\bar{G}: g^{i}=g^{i}(\bar{s})$ be $n_{2}$ Bishop spherical image of $C$. In that case, the following equation

$$
\frac{k_{2}^{2}}{\left(k_{1}^{2}+k_{2}^{2}\right)^{3 / 2}}\left(v_{1}^{k} \dot{\nabla}_{k} \frac{k_{2}}{k_{1}}\right)-v_{1}^{k} \dot{\nabla}_{k} \frac{k_{1} k_{2}}{\sqrt{k_{1}^{2}+k_{2}^{2}}}=\text { constant }
$$

is satisfied.

Theorem 10. Let $\bar{G}: g^{i}=g^{i}(\bar{s})$ be ${ }_{2}$ Bishop spherical image of C. If $\frac{k_{1}}{k_{2}}=$ constant, then $\underset{3}{\bar{v}}$ is parallel translated along $\bar{v}$.

Proof. With reason of equation (72), the following equality can be written:

$$
\begin{aligned}
\bar{v}^{k} \dot{\bar{\nabla}}_{k} \bar{v}^{i}=\underset{31}{\bar{a}^{i}=} & -\frac{k_{1}}{k_{2}^{2}}\left({\underset{1}{k}}^{k} \dot{\nabla}_{k} \frac{1}{\bar{H}_{1}}\right) \boldsymbol{n}_{2}^{i} \\
& -\frac{1}{k_{2}} \frac{1}{\bar{H}_{1}}\left(v_{1}^{k} \dot{\nabla}_{k} \frac{k_{1}}{k_{2}}\right) \underset{2}{n^{i}} \\
& +\frac{1}{k_{2}}\left(v_{1}^{k} \dot{\nabla}_{k} \frac{1}{\bar{H}_{1}}\right)_{1}^{n^{i}}
\end{aligned}
$$

Taking the prolonged covariant derivative of $\frac{1}{\bar{H}_{1}}$ in the direction of $v$, we get

$$
v_{1}^{k} \dot{\nabla}_{k} \frac{1}{\bar{H}_{1}}=-\frac{k_{1} k_{2}^{2}}{\left(k_{1}^{2}+k_{2}^{2}\right)^{3 / 2}}\left(v_{1}^{k} \dot{\nabla}_{k} \frac{k_{1}}{k_{2}}\right)
$$

Using (76) in (75), under the condition $\frac{k_{1}}{k_{2}}=$ constant, we obtain

$$
\underset{31}{\bar{a}^{i}}=\mathbf{0} .
$$

So, we see that $\underset{3}{\bar{v}}$ is parallel translated along $\underset{1}{\bar{v}}$.

\section{Conclusions}

In this work, we have defined some special curves, such as general helix, slant helix, spherical curve and circle in Weyl space, by using prolonged covariant derivative. Later, we have examined the spherical images of a curve and the conditions to be special curves of these images. Further, we expressed the relations among the vector fields of Frenet-Serret and Bishop frame belonging to the images. We have seen that, all of the concepts discussed can be written in terms of Bishop curvatures. Earlier, the equivalents in Weyl space of Bishop curvatures were obtained. Thus, they were used in this discussion. Finally, parallel displacement condition for binormal vector fields of $n_{1}$ and $n_{2}$ spherical images was formulated.

\section{Acknowledgement}

The author would like to thank the anonymous reviewers for providing very insightful and constructive comments.

\section{References}

Bishop, R. L. (1975). There is more than one way to frame a curve. The American Mathematical Monthly, 82(3), 246-251.

Bükçü, B., \& Karacan, M. K. (2007). The Bishop Darboux rotation axis of the spacelike curve in Minkowski 3-space. Ege University Journal of Faculty of Science, 3(1), 1-5.

Bükçü, B., \& Karacan, M. K. (2008a). Special Bishop motion and Bishop Darboux rotation axis of the space curve. Journal of Dynamical Systems and Geometric Theories, 6(1), 27-34.

Bükçü, B., \& Karacan, M. K. (2008b). On the slant helices according to Bishop frame of the timelike curve in Lorentzian space. Tamkang Journal of Mathematics, 39(3), 255-262.

Bükçü, B., \& Karacan, M. K. (2009). The slant helices according to Bishop frame. International Journal of Computational and Mathematical Sciences, 3(2), 67-70.

Izumiya, S., \& Takeuchi, N. (2004). New special curves and developable surfaces. Turkish Journal of Mathematics, 28(2), 153-164.

Karacan, M. K., \& Bükçü, B. (2008). Bishop frame of the timelike curve in Minkowski 3-space. Suleyman Demirel University Journal of Science, 3(1), 80-90.

Kofoğlu, N. (2020). Slant Helices According to Type-1 Bishop Frame in Weyl Space. International Mathematical Forum 15(4), 163-171.

Kula, L., Ekmekci, N., Yaylı, Y., \& İlarslan, K. (2010). Characterizations of slant helices in Euclidean 3-space. Turkish Journal of Mathematics, 34(2), 261-274.

Nomizu, K. \& Yano, K. (1974). On circles and spheres in Riemannian Geometry. Mathematische Annalen, 134, 163170. 
Özdeğer, A. \& Şentürk, Z. (2002). Generalized circles in Weyl spaces and their conformal mapping. Publ. Math. Debrecen, 60, 75-87.

Şemin, F. (1983). Differential Geometry I. İstanbul University.

Tsareva, B., \& Zlatanov, G. (1990). On the geometry of the nets in the n-dimensional space of Weyl. Journal of Geometry, 38(1-2), 182-197.

Yilmaz, S. (2009). Position vectors of some special spacelike curves according to Bishop frame in Minkowski space $\boldsymbol{E}_{\mathbf{1}}^{\mathbf{3}}$. Scientia Magna, 5, 47-49.

Yılmaz, S., Özyllmaz, E., \& Turgut, M. (2010). New spherical indicatrices and their characterizations. Analele Ştiinţifice ale Universităţii Ovidius, 18(2), 337-354. 